\title{
EFFECTS OF FAILURE OF THE RIGHT SIDE OF THE HEART AND INCREASED PULMONARY RESISTANCE ON MECHANICAL CIRCULATORY SUPPORT WITH USE OF THE MINIATURIZED HIA-VAD DISPLACEMENT PUMP SYSTEM
}

Ferdinand Waldenberger, MD

Yong-In Kim, MD

Sarra Laycock, $\mathrm{PhD}$

Bart Meyns, MD

Willem Flameng, $\mathrm{MD}, \mathrm{PhD}$
This experimental study was designed to assess the influence of failure of the right side of the heart or pulmonary hypertension, or both, on the performance of a novel miniaturized left ventricular assist device. In small-sized dogs $(n=$ 50) ischemic global left ventricular failure was induced and support was provided by the HIA-VAD displacement pump (stroke volume 10 or $25 \mathrm{ml}$ ) installed as a left ventricular assist device. In three groups of animals $(n=10$ each) pulmonary hypertension was created before induction of global left ventricular failure. During left ventricular assist device support temporary ischemic failure of the right side of the heart was induced in four groups of animals ( $n=10$ each). In the group subjected to left ventricular failure, support with the left ventricular assist device, and right ventricular failure during left ventricular assist, left atrial pressure and cardiac index were significantly lower than in the group subjected to left ventricular failure and left ventricular assist alone ( $2 \pm 6$ versus $11 \pm 6 \mathrm{~mm} \mathrm{Hg}$ and $1.6 \pm 0.4$ versus $1.0 \pm 0.4 \mathrm{~L} /\left(\mathrm{min} / \mathrm{m}^{2}\right)$, respectively, $\left.p<0.05\right)$. In the group subjected to pulmonary hypertension, left ventricular failure, and left ventricular support, Ieft atrial pressure dropped to values near zero but cardiac index remained unaltered as compared with results with the same regimen without pulmonary hypertension. However, when right ventricular failure was added (that is, pulmonary hypertension, left ventricular failure, left ventricular support, and right ventricular failure during support with the left ventricular assist device) left atrial pressure dropped to negative values $(p<0.05)$ and cardiac index progressively deteriorated. When, in an additional group of dogs, biventricular support was installed in the latter regimen, circulation was initially well supported but oxygenation deteriorated in $60 \%$ of cases. We conclude that (1) adequate right ventricular function was indispensable during support with the left ventricular assist device, (2) the combination of pulmonary hypertension and right ventricular failure led to the "low left ventricular assist device output syndrome," and (3) biventricular mechanical support in the presence of pulmonary hypertension may be complicated by the alveolar leakage syndrome. (J Thorac Cardiovasc Surg 1996;112:484-93)
D splacement pumps that are routinely used for assisted circulation have a common disadvantage, that is, their large pump chamber size. The

From the Department of Cardiac Surgery, Katholieke Universiteit Leuven, Leuven, Belgium.

Received for publication June 7, 1995; revisions requested August 8, 1995; revisions received Oct. 25, 1995; accepted for publication Oct. 30, 1995.

Address for reprints: W. Flameng, MD, PhD, C.E.H.A., Provisorium I, Minderbroedersstraat 17, B-3000 Leuven, Belgium.

Copyright (C) 1996 by Mosby-Year Book, Inc.

$0022-5223 / 96 \$ 5.00+0 \quad \mathbf{1 2 / 1 / 7 0 6 0 4}$
Pittsburgh transplant group quite correctly concluded in its report about pediatric cardiac transplantation that the lack of an efficient miniaturized ventricular assist device is a glaring deficiency in the armamentarium of the pediatric cardiac transplant surgeon. ${ }^{1}$

Indeed, in children or infants the limitations of displacement pumps become obvious when an adult-sized pump is used. The pump rate in small patients has to decrease greatly; otherwise the filling remains incomplete and the rate of thromboembolic complications increases dramatically. A solution to this problem could be miniaturization of the ventric- 
ular assist device (VAD). The HIA-VAD, designed by the Helmholtz Institute at Aachen, Germany, is a displacement pump that can be miniaturized as a result of several new features in design and manufacturing. A miniaturized version became available to us and was used in this experimental study. ${ }^{2}$

Furthermore, in pediatric cardiac operations, the problem of low cardiac output syndrome as a result of failure of the left, right, or combined left and right sides of the heart is often associated with pulmonary vasoactive crises, which are more common than in adults. ${ }^{3}$ It is therefore necessary to study a pediatric assist device in this complex pathophysiologic setting. To study the effect of failure of the right side of the heart and pulmonary hypertension on support with a left ventricular assist device (LVAD), we designed an experimental animal model of severe isolated global failure of the left side of the heart. Once left ventricular failure (LVF) was induced, an LVAD was connected and the circulation was supported by the pediatric HIA-VAD system. In a second protocol, temporary failure of the right side of the heart was induced during LVAD support for LVF, and its influence on LVAD function was demonstrated. In this way "low LVAD output syndrome" could be studied. In a third protocol, the influence of acute pulmonary hypertension on the low LVAD output syndrome was assessed. Here, acute pulmonary hypertension was created in a series of animals, LVF was induced and the circulation supported by an LVAD. By comparison of the results with those obtained in animals without pulmonary hypertension, the effect of increased pulmonary vascular resistance on LVAD output could be studied. In another protocol, the influence of the combination of right ventricular failure (RVF) and pulmonary hypertension on LVAD output could be studied. Finally, the influence of use of a biventricular assist device (BVAD) was demonstrated under these circumstances.

\section{Material and methods}

Experimental preparation. Fifty-five mongrel dogs with a body weight of 6 to $18 \mathrm{~kg}$ were used in this study. In dogs the right coronary artery exclusively supplies the right ventricle and only a small part of the anterior wall of the right ventricle is perfused by the left anterior descending artery. Selective occlusion of left or right coronary arteries makes the dog a suitable experimental species for isolated failure of the right or left side of the heart.

All animals received humane care in compliance with the "Guide for the Care and Use of Laboratory Animals" formulated by the National Academy of Sciences and published by the National Institutes of Health (NIH Publication No. 85-23, revised 1985) and with the guidelines of the local ethical committee of the Katholieke Universiteit Leuven.

The dogs were premedicated with Hypnorm analgesic (fluanisone $10 \mathrm{mg}$ and fentanyl $0.2 \mathrm{mg} / \mathrm{ml}$ ) $0.2 \mathrm{ml} / \mathrm{kg}$ body weight intramuscularly and anesthetized with Nembutal anesthetic, $0.2 \mathrm{ml} / \mathrm{kg}$ (sodium pentobarbital $60 \mathrm{mg} / \mathrm{ml}$ ), administered intravenously. The dogs were then intubated and the lungs mechanically ventilated with a mixture of oxygen, room air, and halothane, $0.2 \%$ to $0.4 \%$ (until instrumentation was completed). Ventilation was adjusted to maintain $\mathrm{pH}$ and oxygen levels in the satisfactory range as indicated by determination of arterial blood gas values. Routine respiration rate was 20 breaths/min, tidal volume was 18 to $20 \mathrm{ml} / \mathrm{kg}$ body weight, and positive end-expiratory pressure $5 \mathrm{~cm} \mathrm{H}_{2} \mathrm{O}$. Additional doses of Dipodolor analgesic (piritramide $10 \mathrm{mg} / \mathrm{ml}$ ) and Rapifen analgesic (fentanyl $0.5 \mathrm{mg} / \mathrm{ml}$ ) were administered as required to maintain anesthesia.

After induction of anesthesia, catheters were inserted into the right femoral artery for obtaining arterial pressures and blood samples and into the right femoral vein for blood transfusions from donor dogs or autologous blood retransfusions. In dogs with a hemoglobin content of more than $12.5 \mathrm{gm} / \mathrm{dl}$ an isovolumic hemodilution was done before the thoracotomy to obtain approximately 200 $\mathrm{ml}$ autologous blood.

A catheter was placed in the left jugular vein for measurements of right atrial pressure and for injecting ice-cold saline solution for cardiac output measurements. Furthermore, a urinary catheter was directly inserted into the urinary bladder through a midline minilaparotomy.

After administration of a $0.2 \mathrm{ml} / \mathrm{kg}$ dose of Pavulon muscle relaxant (pancuronium $2 \mathrm{mg} / \mathrm{ml}$ ), a thoracotomy was done through the fifth intercostal space. An 8 or 10 $\mathrm{mm}$ Intravascular graft (for the HIA-VAD $10 \mathrm{ml}$ or $25 \mathrm{ml}$, respectively) was anastomosed end to side to the descending thoracic aorta during partial clamping. $A$ bolus of lidocaine (Xylocaine; $1 \mathrm{mg} / \mathrm{kg}$ ) was injected and a continuous intravenous infusion started at a rate of $1 \mathrm{mg} / \mathrm{kg}$ per hour before the pericardium was opened and the heart suspended in a pericardial cradle. The coronary arteries were dissected (the left anterior descending and the left circumflex coronary artery distally from the main stem and the right coronary artery) and snares were placed around them. According to the protocol, occlusion was produced by tightening the snare with a tourniquet and reperfusion was recognized as the appearance of reactive hyperemia after removal of the snare.

During the biventricular pumping an 8 or $10 \mathrm{~mm}$ vascular graft was anastomosed end to side to the main pulmonary artery during partial clamping.

After systemic heparinization with a heparin dose of 100 to $200 \mathrm{IU} / \mathrm{kg}$, depending on activated clotting times, a balloon-tipped catheter (Swan-Ganz catheter, Baxter Healthcare Corp., Edwards Division, Santa Ana, Calif.) was placed in the left pulmonary artery through a stab incision in the right ventricular outflow tract. Activated clotting time was maintained in the range of 150 to 200 seconds and measured with a Hemochron 400 instrument (International Technidyne Corp., Edison, N.J.). Further, a 


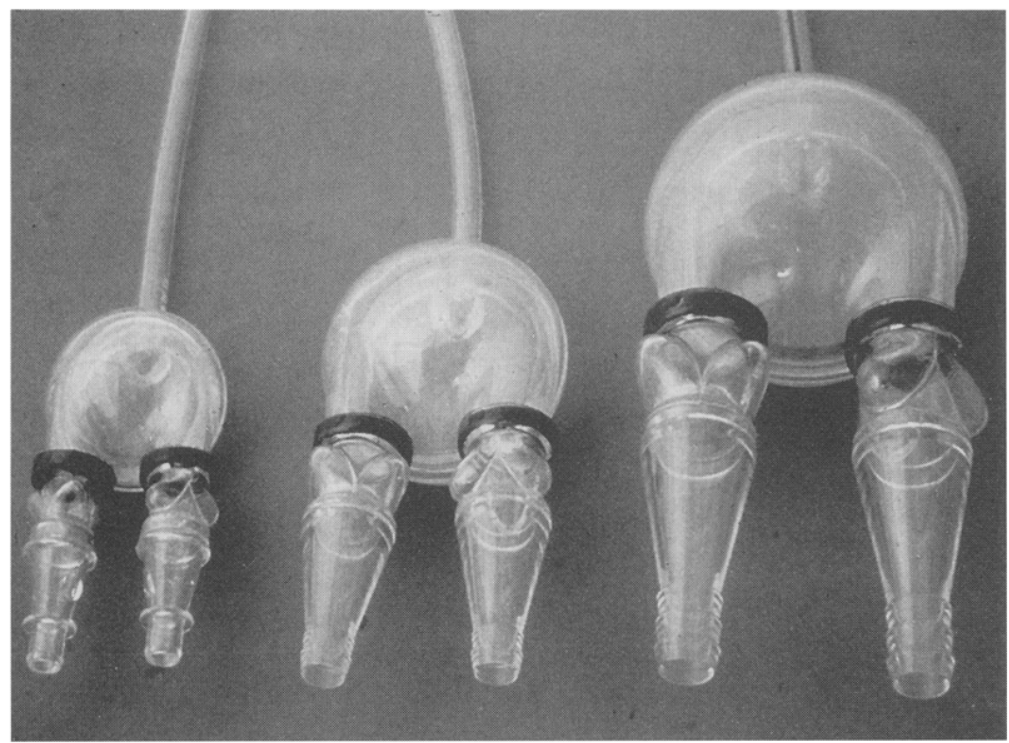

Fig. 1. Photograph of HIA-VAD pumps. Devices available had stroke volumes of $10 \mathrm{ml}, 25 \mathrm{ml}$, or $60 \mathrm{ml}$.

catheter was inserted into the left ventricle through a stab incision in the apex for left ventricular pressure recordings.

The HIA-VAD pump. The HIA-VAD displacement pump is a pneumatically driven membrane-type pump ${ }^{2}$ (Fig. 1), of which by different sizes with varying pump volumes can be manufactured. Besides the currently available $60 \mathrm{ml}$ (adult size) pump, two miniaturized versions for pediatric use were produced and were used in our studies. These versions have maximal stroke volumes of 10 $\mathrm{ml}$ and $25 \mathrm{ml}$, respectively. For biventricular support the right-sided pumps are available with $10 \%$ less stroke volume compared with that of left-sided pumps. This smaller stroke volume on the right side was achieved by decreasing the diameter of the pump membrane. Thus different pump outputs can be achieved although the two pumps operate at the same pump rates.

The artificial valves have three leaflets and sinuses, with the valves and outlet being conus shaped. This structure was based on findings obtained from in vitro flow visualization techniques and finite element method stress analysis, which showed the conical design to have superior performance especially in the open position. ${ }^{2}$ This new valve design allows a reduction of driving pressures and vacuum, improves the filling behavior, and avoids clot formation as demonstrated during in vivo testing. ${ }^{2}$ With use of a new manufacturing technique, the valves are made of polyurethane with favorable biocompatibility features. Because of the unique molding process, a pump is produced that is seamless and has optimal membrane performance even in the smallest available size of $10 \mathrm{ml}$ pumping volume. A special gluing technique provides a smooth transition between the parts, and silicone oil used as a lubricant between the pump membranes leads to a completely transparent blood pump that can be easily deaired. All surfaces in contact with blood were made of biocompatible polyurethane.

A new driving system manufactured by Medos (Medizintechnik GmbH, Stolberg, Germany) was used. This system uses compressed air and applies vacuum and positive pressure. Four different parameters can be altered and adjusted according to the hemodynamic needs: systolic driving pressure, diastolic vacuum, duration of systole, and pump rate. This unit can provide univentricular and biventricular support for all three pump sizes.

The $10 \mathrm{ml}$ HIA-VAD pump was used in dogs with a body weight between 6 and $10 \mathrm{~kg}(n=25)$, and the $25 \mathrm{ml}$ HIA-VAD pump was used in dogs with a body weight between 10 and $19 \mathrm{~kg}(n=25)$. In the control group without HIA-VAD pump support $(n=5)$ the body weight was $12 \pm 1 \mathrm{~kg}$.

The HIA-VAD pump was connected to the inflow cannula (Polystan Bent Hard No. 28 [Polystan, Copenhagen, Denmark] in case of HIA-VAD $10 \mathrm{ml}$ pump or No. 36 in case of HIA-VAD $25 \mathrm{ml}$ pump), which was introduced into the left auricular appendage and secured with a purse-string suture, and to the outflow cannula (Research Medical FEM FLEX No. 20 or No. 24 [Research Industries Corp., Midvale, Utah], respectively), which was inserted into the graft on the descending aorta. A catheter was inserted into the left atrium.

Depending on the protocol the right ventricular assist device (RVAD) was connected to the inflow cannula (Polystan Bent Hard No. 28 or No. 36), which was positioned in the right atrium after being introduced into the right auricular appendage through a purse-string suture, and to the outflow cannula (Research Medical FEM FLEX No. 20 or No. 24), which was inserted into the graft in the pulmonary artery. If the animals could be weaned from the VADs the cannulas were removed. After 
the experiment the animals were killed by an intravenous injection of pentobarbital and potassium chloride.

Experimental protocols. The canine coronary system is strongly collateralized. ${ }^{4}$ Therefore the LVF model required several subsequent coronary occlusions.

Global LVF was induced in all dogs. This was done by sequential occlusions of the left anterior descending artery ( 20 minutes) and the circumflex artery ( 45 minutes) followed by reperfusion. Circumflex occlusion was done after 5 minutes of reperfusion of the left anterior descending artery. This sequence induced severe global left ven tricular stunning (LVF). LVAD support was started shortly after circumflex occlusion, that is, when severe LVF occurred. According to the protocol, RVF was induced after LVAD support was begun in the presence of LVF. RVF was induced by 45 minutes of occlusion of the right coronary artery followed by reperfusion.

Acute pulmonary hypertension was produced by injection of $100 \mu \mathrm{m}$ glass beads into the pulmonary circulation $^{5,6}$ before LVF was induced.

The following experimental protocols were designed.

Groups without pulmonary hypertension. Three groups had no pulmonary hypertension: (1) LVF with no mechanical. support (group I, $n=5$ ); (2) LVF plus LVAD support (group II, $n=10$ ); and (3) LVF plus LVAD support plus RVF during LVAD support (group III, $n=$ $10)$.

Groups with pulmonary hypertension. Three further groups with pulmonary hypertension were formed: (1) LVF plus LVAD support (group IV, $n=10$ ); (2) LVF plus LVAD support plus RVF during LVAD support (group V, $n=10$ ); and (3) LVF plus LVAD support plus RVF and RVAD support (group VI, $n=10$ ).

Systolic, diastolic, and mean systemic pressures and pulmonary arterial pressure (PAP), left atrial pressure (LAP), right atrial pressure (RAP), maximum and minimum rate of pressure rise, cardiac output, mixed venous blood gas values, and urinary production were measured at baseline before ischemia; after 10 and 19 minutes of occlusion of the left anterior descending artery; after 5 minutes of reperfusion; after 10, 20,30, and 40 minutes of circumflex occlusion; after $5,10,15,30,45$, and 60 minutes of reperfusion; and then every 30 minutes until the end of the experiment. Blood samples for lactate determination were taken at baseline, and sampling was restarted at reperfusion after circumflex occlusion at the intervals described.

Statistics. All data are expressed as mean values plus or minus standard deviations. The differences within one experiment and the differences between individuals and groups were computed with a two-factor (time and treatment) analysis of variance and with repeated measures on one factor (time). If the $F$ value was significant, the differences between groups were evaluated with Fisher's test as a post hoc test, considering a probability value of less than $5 \%(p<0.05)$ to be significant.

The significance of changes from baseline values was determined by the Bonferroni-Dunn test.

For the computations we used the commercially available software programs StatView 4.0 (Abacus Concepts, Inc., Berkeley, Calif.) and SuperANOVA 1.11 (Abacus
Concepts) on a MacIntosh Powerbook 180 (Apple Computer, Inc., Cupertino, Calif.).

\section{Results}

Validation of the experimental model. In the control group (LVF without LVAD) irreversible cardiogenic shock developed during global left ventricular dysfunction in all animals. After induction of anterior wall akinesia by occlusion and reperfusion of the left anterior descending artery, cardiac index dropped from $3.8 \pm 1$ to $2.6 \pm 1.4 \mathrm{~L} /\left(\mathrm{min} / \mathrm{m}^{2}\right)(p<$ $0.05)$ and LAP increased from $5 \pm 4 \mathrm{~mm} \mathrm{Hg}$ to $13 \pm$ $10 \mathrm{~mm} \mathrm{Hg}(p<0.05)$. Also, mean aortic pressure decreased from $84 \pm 21$ to $65 \pm 20 \mathrm{~mm} \mathrm{Hg}$, mean pulmonary pressure increased from $12 \pm 3$ to $19 \pm$ $9 \mathrm{~mm} \mathrm{Hg}$, and RAP also increased from $4 \pm 2$ to $8 \pm$ $6 \mathrm{~mm} \mathrm{Hg}$. These changes, however, did not reach statistical significance $(p>0.05)$.

This part of the experimental protocol was similar in all further groups and the data obtained in the other groups did not differ significantly from those in the control group $(p>0.05$ ). Subsequent occlusion and reperfusion of the circumflex artery, that is, after reperfusion of the left anterior descending artery, induced global left ventricular hypocontractility. Ventricular fibrillation necessitating frequent defibrillation attempts and cardiac massage also occurred during this phase. Progressively, irreversible low cardiac output developed and four of five dogs died within the first hour of global LVF. One dog initially recovered but died 2 hours after induction of global LVF as a result of low cardiac output and intractable ventricular fibrillation.

Effect of RVF on LVAD support. When LVAD support was started during global LVF (group II), hemodynamics stabilized and all animals could be weaned from LVAD support after 90 minutes of support. During the last 45 minutes of LVAD support, it should be noted, all coronary arteries were reopened. During this period the LVAD compensated for postischemic global left ventricular dysfunction as observed in group I and allowed recovery from global stunning. All animals survived the observation period.

During LVAD support, LAP progressively dropped from $15 \pm 7 \mathrm{~mm} \mathrm{Hg}$ to $8 \pm 1 \mathrm{~mm} \mathrm{Hg}$ at the end of support ( $p<0.05$; Fig. 2, lower left panel). Mean aortic pressure and cardiac index recovered to prepump levels at the end of support (Fig. 3, left panels, triangles). Mean PAP and RAP decreased during LVAD support to baseline values (Fig. 2, upper left panel). 


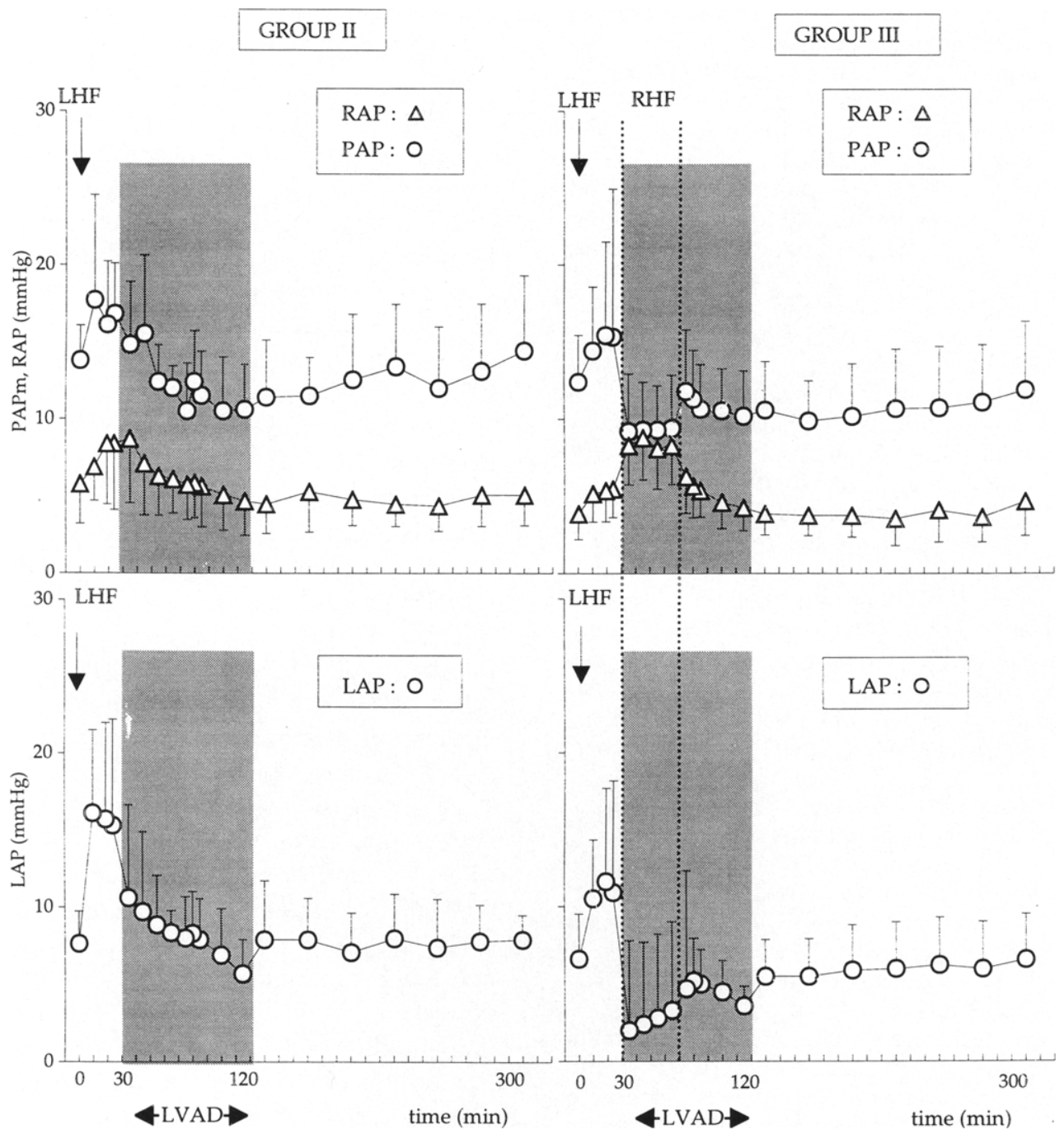

Fig. 2. Hemodynamics before, during (shaded area), and after LVAD support. Arrows indicate induction of failure of left side of heart $(L H F)$ or failure of right side of heart $(R H F)$. $m P A P$, Mean PAP.

On induction of transient RVF during LVAD (group III), PAP dropped significantly, RAP increased significantly $(p<0.05)$, and both pressures equalized (Fig. 2, upper right panel). At the same time, LAP decreased to much lower values than during the same interval in group II (LHF plus LVAD without RHF): $11 \pm 6 \mathrm{~mm} \mathrm{Hg}$ in group II versus $2 \pm 6 \mathrm{~mm} \mathrm{Hg}$ in group III $(p<0.05$; Fig. 2, lower right panel). Simultaneously, the cardiac index dropped to a significantly lower value than in group II $\left(1.6 \pm 0.4 \mathrm{~L} /\left[\mathrm{min} / \mathrm{m}^{2}\right]\right.$ in group II versus $1 \pm 0.4$ $\mathrm{L} /\left[\mathrm{min} / \mathrm{m}^{2}\right]$ in group III; $p<0.05$ ) (Fig. 3, lower left panel).

After reperfusion of the right ventricle during LVAD support, right ventricular function recov- ered, PAP increased, RAP decreased, and LAP and cardiac index increased to levels comparable to those in group II ( $p>0.05$; Fig. 2, right panels). Also, hemodynamics after weaning did not differ significantly between the groups $(p>0.05)$. The data are presented in Figs. 2 and 3.

Effect of pulmonary hypertension on LVAD support. The injection of glass beads resulted in a significant increase in pulmonary resistance and PAP. Pulmonary resistance was $613 \pm 222 \mathrm{~mm}$ $\mathrm{Hg} \cdot \mathrm{min} \cdot \mathrm{m}^{2} / \mathrm{L}$ and $538 \pm 160 \mathrm{~mm} \mathrm{Hg} \cdot \mathrm{min} \cdot \mathrm{m}^{2} / \mathrm{L}$ in groups IV and $\mathrm{V}$, respectively, versus $138 \pm 45$ $\mathrm{mm} \mathrm{Hg} \cdot \mathrm{min} \cdot \mathrm{m}^{2} / \mathrm{L}$ and $164 \pm 107 \mathrm{~mm}$ $\mathrm{Hg} \cdot \mathrm{min} \cdot \mathrm{m}^{2} / \mathrm{L}$ in groups II and III, respectively $(p<0.05)$. Mean PAP was $27.6 \pm 4 \mathrm{~mm} \mathrm{Hg}$ and 


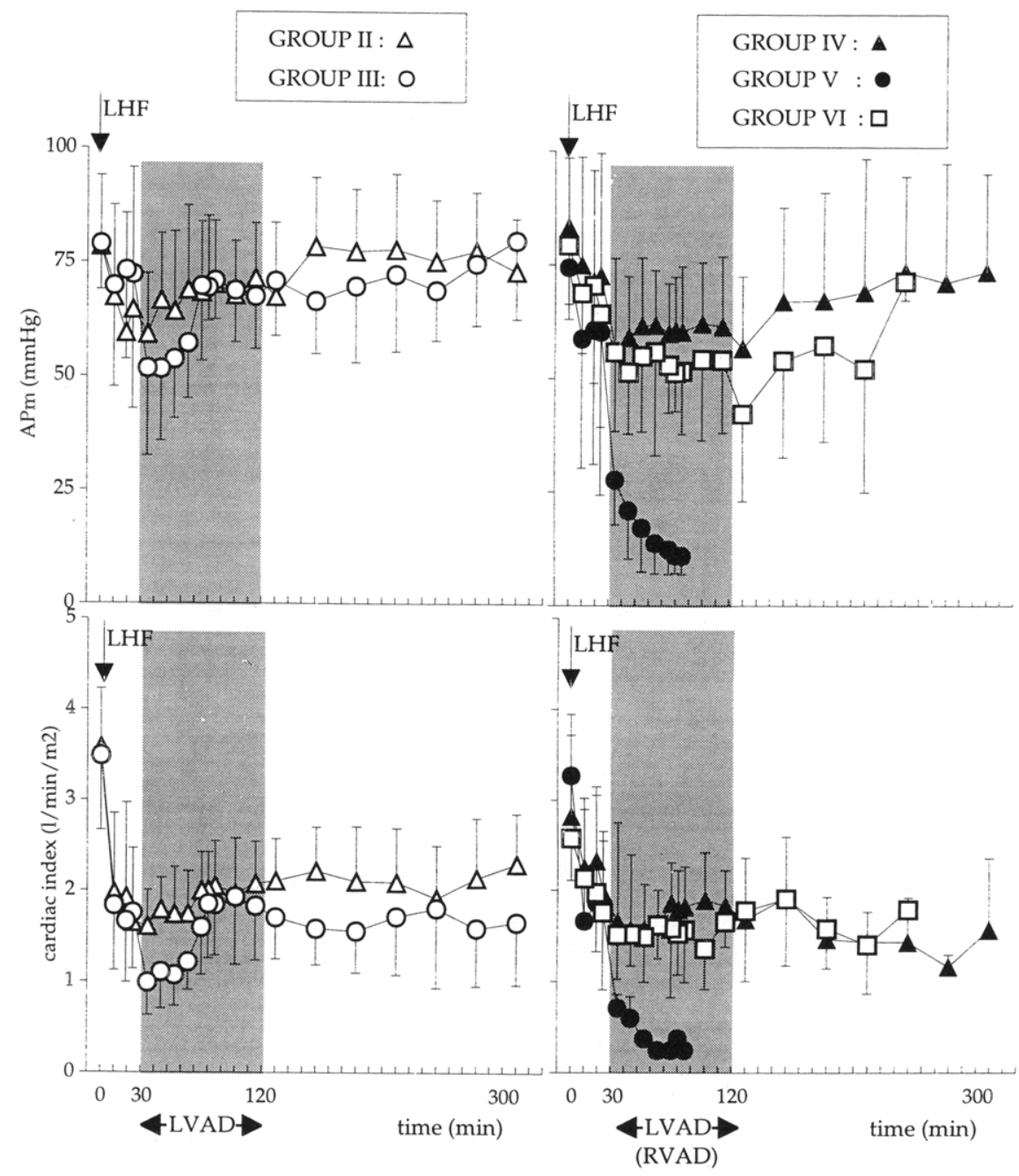

Fig. 3. Hemodynamics before, during (shaded area), and after LVAD support. In groups III, IV, and VI RVF was induced after LVF, as indicated in Fig. 2, right panels. In group VI biventricular support was performed. $A P m$, Mean aortic pressure; $L H F$, failure of left side of heart.

$26.9 \pm 3.5 \mathrm{~mm} \mathrm{Hg}$ in groups IV and $\mathrm{V}$, respectively, versus $13.8 \pm 2.2 \mathrm{~mm} \mathrm{Hg}$ and $12.4 \pm 2.9 \mathrm{~mm} \mathrm{Hg}$ in groups II and III, respectively $(p<0.05)$.

When LVF was induced in groups IV and $V$, hemodynamic changes occurred comparable to those in groups II and III, with the difference that the already increased PAP decreased slightly but not significantly.

Installation of LVAD support in group IV (Fig. 4, lower left panel) resulted in an immediate decrease of LAP to zero pressure during the entire LVAD pumping period. This clearly contrasted with the corresponding data in group II $(p<0.01)$. PAP dropped slightly with the onset of LVAD support but remained substantially higher than RAP throughout the time of pump support (Fig. 4, upper left panel). Mean arterial pressure and cardiac index during LVAD support was somewhat lower than in group II although the difference did not reach significance $(p>0.05$; Fig. 3, closed triangles, right panels).

After weaning from the LVAD, cardiac index and arterial pressure slowly deteriorated, and 3 of the 10 animals died as a result of low cardiac output.

When transient RVF was induced during LVAD support in animals having pulmonary hypertension (group V), PAP dropped dramatically to $8 \mathrm{~mm} \mathrm{Hg}$ and equalized with RAP (Fig. 4, upper right panel). LAP dropped immediately to negative values $(p<$ 0.05) (Fig. 4, lower right panel). Further, arterial 


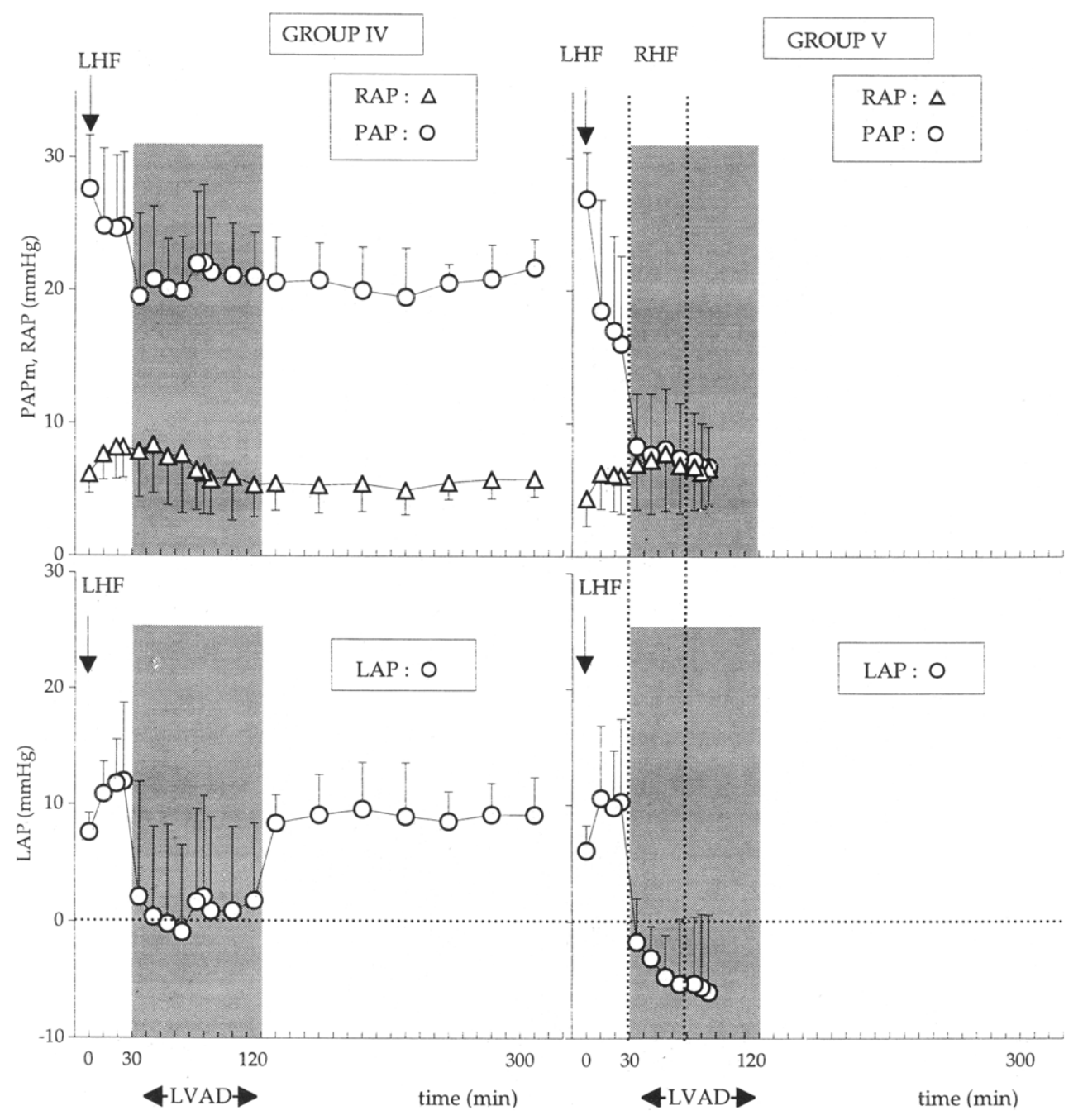

Fig. 4. Hemodynamics before, during (shaded area), and after LVAD support. Abbreviations are same as used in Fig. 2.

pressure and cardiac index dropped sharply to zero. Reperfusion of the right ventricle did not result in any recovery of LVAD output and cardiac index dropped toward zero (Fig. 3, closed circles, right panel). Thus irreversible low LVAD output syndrome developed and all animals died during LVAD support.

Biventricular HIA-VAD pump support. In group VI animals, pulmonary hypertension was also induced whereby mean PAP increased to $27.9 \pm 6 \mathrm{~mm}$ $\mathrm{Hg}$. LVF was induced as in all previous protocols and hemodynamics changed initially in a way similar to those in protocols IV and V. Then RVF was induced and BVAD support was started immediately (Fig. 5) This resulted in a small drop in PAP and RAP but not in an equalization of PAP and
RAP as seen in group V. Pulmonary pressure remained high and RAP decreased during BVAD support (Fig. 5, upper panel). LAP dropped to zero (Fig. 5, lower panel) and cardiac index was stable at about $1.5 \mathrm{~L} /\left(\mathrm{min} / \mathrm{m}^{2}\right)$. In 3 of 10 animals, however, oxygenation deteriorated and fluid exudated into the trachea. In these animals a low BVAD output syndrome occurred and the animals died. In the remaining seven animals, LAP dropped to zero, but cardiac index remained stable (Fig. 3, open squares, lower right panel). In these animals mixed venous oxygenation ranged between $45 \%$ and $75 \%$. After weaning from BVAD support, low cardiac output developed in three further animals and they died during the observation period. Thus 4 of 10 animals survived the entire observation period. 


\section{Discussion}

The pulmonary circuit represents only about $10 \%$ of the total peripheral resistance against which the heart performs, an additional load that is exactly bearable by the left ventricle alone. ${ }^{7}$ In clinical cases of excessive right ventricular infarction, pressures recorded in the pulmonary artery and in the right atrium equalized, indicating that the right ventricle did not serve as a pump any more. Under these conditions, the right ventricle was considered to be dispensable, ${ }^{8}$ because the intact left side of the heart alone could support the circulation.

Our results show, however, that this concept of the dispensable right ventricle is not valid in case of left ventricular mechanical assistance. Most probably the reason for this is related to the importance of the activity of the interventricular septum. Indeed, malfunction of the interventricular septum has a severe impact on right ventricular function,, 910 and it might well be that the right ventricle, defined as the right ventricular free wall, may be dispensable provided the interventricular septum is normocontractile and the pulmonary resistance remains normal. In a situation of global left ventricular dysfunction, although the ventricle is supported and unloaded by an LVAD system, the septal wall motion is lacking. Therefore right ventricular ischemia and dysfunction immediately lead to a "low LVAD output syndrome."

It has been previously reported in an experimental model of acute pulmonary hypertension that the increase in mean pulmonary pressure was associated with a decrease in stroke volume and cardiac output, even under conditions of normal right ventricular function. ${ }^{11} \mathrm{We}$ also observed this phenomenon after induction of pulmonary hypertension during baseline conditions in our experiments: cardiac output was lower than in the groups without pulmonary hypertension. Furthermore, a study on increased right ventricular afterload showed that under normal conditions the right ventricle operates as a flow pump and has reserves in terms of increasing its stroke work. ${ }^{9}$ In this mode if the maximal stroke work occurred at a mean PAP of 20 to $30 \mathrm{~mm} \mathrm{Hg}$, the right ventricle failed as a pump. Decreasing the cardiac output decreases the oxygen supply, and this will further reduce organ function. Right ventricular efficiency will decline and, at even lower pulmonary pressures, pump flow will decrease. The mean PAP at baseline conditions in our experiments was just below $30 \mathrm{~mm} \mathrm{Hg}(27.2 \pm 4.2 \mathrm{~mm} \mathrm{Hg})$, which forced the right ventricle to perform work with the maximal

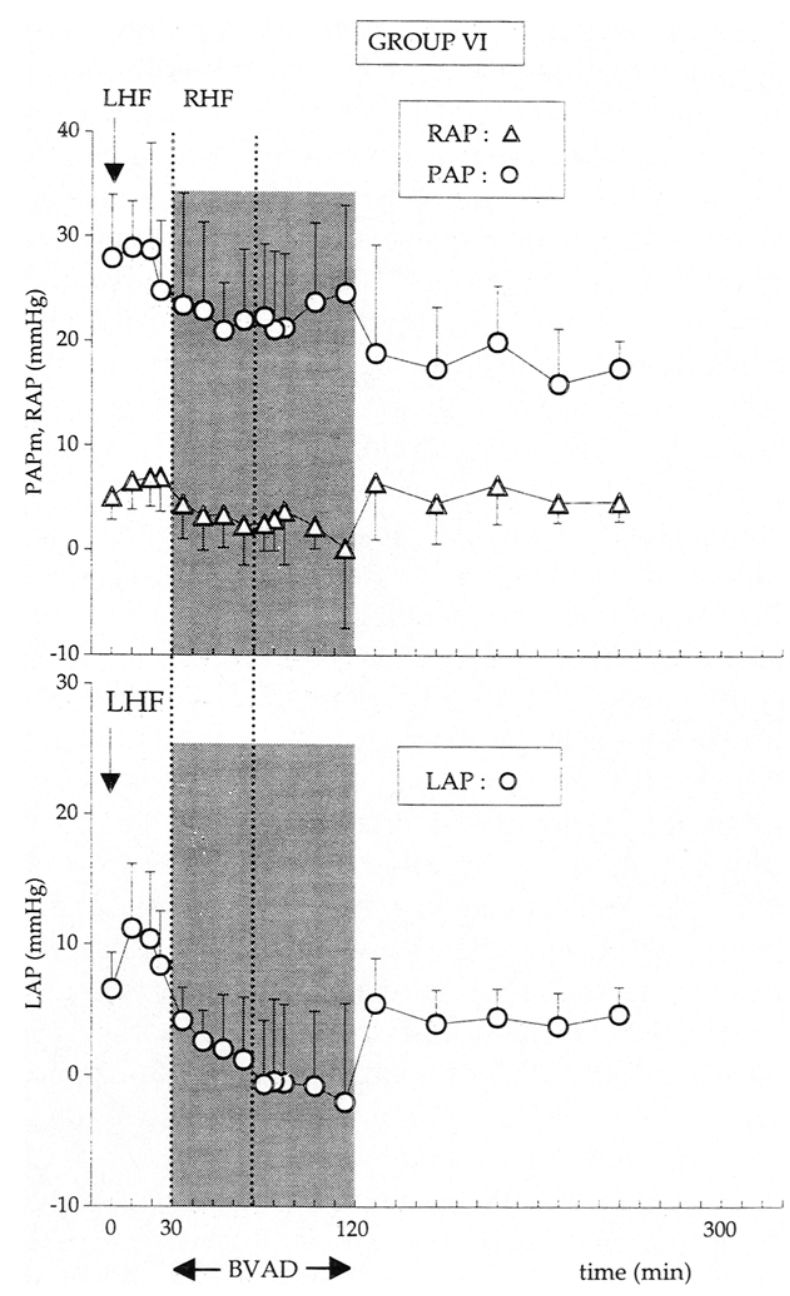

Fig. 5. Hemodynamics before, during (shaded area), and after BVHD. Abbreviations are same as used in Fig. 2.

possible efficiency, provided sufficient oxygen supply was available.

The model of acute pulmonary hypertension produced by glass beads is a well-established model..$^{5,6}$ Glass bead injections produce a situation in the pulmonary microvasculature that is comparable with that of acute respiratory distress syndrome. The amount of pulmonary edema caused by damage of endothelial membranes after glass bead injections is minimal, provided a stable hemodynamic situation is maintained, the right ventricle does not fail, and the gas exchange in the lungs does not deteriorate. Other groups have combined glass bead injections with injections of oleic acid to reach a pulmonary situation that mimics acute respiratory distress syndrome even better and to study gaseous exchange and changes in lung compliance. 
The clinical application of this model is limited to specific situations. Often pulmonary hypertension is reversible and disappears after commencement of left ventricular support. However, in two situations this model of pulmonary hypertension is applicable: (1) fixed pulmonary hypertension in congenital pathologic conditions and (2) pulmonary hypertension as a result of pulmonary edema caused by capillary leak syndrome, which occurs after massive blood transfusion, prolonged bypass time, or acute respiratory distress syndrome.

From our experimental work it is evident that a combination of LVAD support and pulmonary hypertension leads to a decrease in pulmonary blood flow and inflow into the left side of the heart to such an extent that it becomes life threatening. The combination of RVF and pulmonary hypertension was not compatible with survival. As already mentioned, right atrial and pulmonary pressures leveled off during right coronary artery ischemia and LVAD support. After a few minutes of right ventricular ischemia, sufficient inflow into the left side of the heart ceased and the peripheral circulation could not be maintained, regardless of the powerful left ventricular mechanical circulatory support. Even negative left atrial pressures because of maximal vacuum on the left pump did not force enough blood through the lungs.

To overcome this problem of RVF associated with pulmonary hypertension, we added an RVAD to the LVAD and performed biventricular mechanical assisted circulation and mechanical unloading. In the clinical setting the results of such a BVAD support in terms of survival are rather disappointing and hardly reach $40 \%$. The outcome depends largely on patient treatment after biventricular failure has been diagnosed. In patients with biventricular failure who require a BVAD for survival, this decision has to be made quickly. ${ }^{12}$ However, caution is needed in patients with capillary leak syndrome because the acutely increased pulmonary blood flow provided by the RVAD can produce overwhelming pulmonary edema. ${ }^{13} \mathrm{~A}$ suggested way to prevent this is to restrict volume loading in those patients because of the risk of a capillary leak syndrome or to perform hemofiltration. However, these events seem to precede a lethal outcome. ${ }^{14}$ In our experimental series we found a survival rate of $40 \%$, identical to that in the clinical reports. In one dog acute alveolar leakage syndrome developed and acute pulmonary edema led to instant death. In two other dogs progressive edema occurred and the leakage into the tissues proceeded more slowly. The dogs subsequently died after 2 hours.

The development of pulmonary edema in the presence of a very low left atrial pressure is difficult to explain. It might be because acute pulmonary hypertension induces capillary leakage and an increase in extravascular lung water along with ischemia and subsequent release of neurohumoral factors. $^{15}$ The critical closing pressure increases because of progressive arteriolar tension caused by increased extravascular water and humoral factors, ${ }^{16}$ and hypoxemia adds to the impairment. ${ }^{17}$ The critical closing pressure in the lung exceeds LAP and becomes the critical outflow pressure of the right ventricle. ${ }^{18}$ In the group with right coronary artery occlusion and pulmonary hypertension, pulmonary pressures fell under the critical closing pressure and the flow through the lung ceased. However, if RVAD support produced pressures higher than the closing pressures, the flow through the lungs was maintained. Because of the endothelial injury and the overdistention of the pulmonary vessels, high RVAD outflow pressures can induce acute or progressive pulmonary edema as a lethal consequence.

\section{REFERENCES}

1. Armitage JM, Fricker FJ, Nido del P, Starzl TE, Hardesty RL, Griffith BP. A decade (1982 to 1992) of pediatric cardiac transplantation and the impact of FK 506 immunosuppression. J Thorac Cardiovasc Surg 1993;105:464-73.

2. Eilers R, Harbott P, Reul H, Rakhorst G, Rau G. Design improvements of the HIA-VAD based on animal experiments. Int J Artif Organs 1994;18:473-8.

3. Pearson GA, Sosnowsky A, Chan KC, Firmin RK. Salvage of postoperative pulmonary hypertensive crisis using ECMO via cervical cannulation in a case of truncus arteriosus. Eur J Cardiothorac Surg 1993; 7:390-1.

4. Wüsten B, Flameng W, Schaper W. The distribution of myocardial flow: part-effects of experimental coronary occlusion. Basic Res Cardiol 1974;69:422-34.

5. Zwissler B, Forst H, Ishii K, Messmer K. A new experimental model of ARDS and pulmonary hypertension in the dog. Res Exp Med 1989;189:427-38.

6. Delcroix M, Mélot C, Vachiéry JL, et al. Effects of embolus size on hemodynamics and gas exchange in canine embolic pulmonary hypertension. Am Rev Respir Dis 1992;145:80610.

7. Furey SA, Zieske HA, Levy MN. The essential function of the right ventricle. Am Heart J 1984;107:404-10.

8. Sade RM, Castaneda AR. The dispensable right ventricle. Surgery 1975;77:624-31.

9. Vlahakes GJ, Turley K, Hoffman JIE. The pathophysiology of failure in acute right ventricular hypertension: hemodynamic and biochemical correlations. Circulation 1981;63:8795.

10. Spotnitz HM, Berman MA, Epstein SE. Pathophysiology and 
experimental treatment of acute pulmonary embolism. Am Heart J 1971;82:511-20.

11. Fourie PR, Coetzee AR, Bolliger CT. Pulmonary artery compliance: its role in right ventricular-arterial coupling. Cardiovasc Res 1992;26:839-44.

12. Pennington DG, Merjavy JP, Swartz MT, et al. The importance of biventricular failure in patients with postoperative cardiogenic shock. Ann Thorac Surg 1985;39:16-26.

13. Dembitsky WP, Daily PO, Raney AA, Moores WY, Joyo CI. Temporary extracorporeal support of the right ventricle. J Thorac Cardiovasc Surg 1986;91:518-25.

14. Pierce WS. Management of secondary organ dysfunction. Ann Thorac Surg 1993;55:222-6.
15. Hachenberg T, Meyer J, Brüssel T, et al. Effective pulmonary capillary pressure in experimental myocardial ischemia. Eur Heart J 1993;14:705-11.

16. Malik AB, van der Zee H. Mechanism of pulmonary edema induced by microembolization in dogs. Circ Res 1978;42: 72-9.

17. Boitreau P, Ducas J, Schick U, Girling L, Prewitt RM. Pulmonary vascular pressure-flow relationship in canine oleic acid pulmonary edema. Am J Physiol 1986;251: H1163-70.

18. Leeman M, Lejeune P, Closset J, Vachiéry JL, Mélot C, Naeije R. Nature of pulmonary hypertension in canine oleic acid pulmonary edema. J Appl Physiol 1990;69:293-8. 